\section{PATIENTS', RELATIVES' AND HEALTH PROFESSIONALS' DIRECT EXPERIENCES AND PERCEPTIONS OF BISPECTRAL INDEX (BIS) MONITORING IN A UK HOSPICE: A QUALITATIVE INTERVIEW STUDY FOR I-CAN-CARE}

${ }^{1}$ Anna-Maria Krooupa, ${ }^{1}$ Bella Vivat, ${ }^{2}$ Stephen McKeever, ${ }^{1}$ Patrick Stone. ${ }^{1}$ Marie Curie Palliative Care Research Department, University College London, UK; ${ }^{2}$ Department of Advanced and Integrated Practice, London South Bank University, UK

\subsection{6/spcare-2019-mariecuriepalliativecare.31}

Background Research interest is growing in using EEG-based tools, such as Bispectral Index (BIS) technology, to assess levels of sedation for palliative care patients. To date, little research has investigated using BIS in palliative care practice, but previous exploratory work for our I-CANCARE programme asked clinicians, patients, and relatives whether using BIS in palliative care would be acceptable in principle.

Aim To explore the direct experiences and perceptions of palliative care patients, their relatives, and clinicians regarding BIS monitoring in a UK hospice.

Methods We trialled the use of BIS with hospice inpatients, and then conducted semi-structured interviews with them, their relatives, and hospice clinicians. Interview transcripts were analysed following the framework method.

Results Ten patients, two relatives, and ten clinicians participated in individual interviews. Most (as in our previous hypothetical study) considered BIS monitoring non-intrusive. Some patients commented that after the sensor had been applied to their foreheads they were 'not aware' of it. Patients and clinicians commented that, although noticeable, the BIS device and sensor were small and easily handled, and felt that the monitoring did not affect patients' daily routines, nor care activities. After trialling BIS monitoring, participants said that they would have no objections to BIS being used as part of routine care, provided that it was beneficial for patients.

Conclusions Our participants perceived BIS technology as acceptable and feasible for monitoring hospice inpatients' consciousness levels. This indicates that further exploration of how this technology might contribute to palliative care practice would be appropriate, and valuable.

\section{THE TWO SIDES TO COUGH STORIES AND THE TRUTH IN BETWEEN: A QUALITATIVE STUDY OF IDIOPATHIC PULMONARY FIBROSIS (IPF) PATIENTS AND THEIR CLOSEST FAMILY MEMBERS}

${ }^{1}$ Kirsty Hett, ${ }^{1}$ Mirella Longo, ${ }^{2}$ Benjamin Hope-Gill, ${ }^{1}$ Annmarie Nelson. ${ }^{1}$ Cardiff University, UK; ${ }^{2}$ Cardiff and Vale University Health Board, UK

\subsection{6/spcare-2019-mariecuriepalliativecare.32}

Background Idiopathic Pulmonary Fibrosis (IPF) is a progressive condition of the lung with no known cause or cure. Whilst treatments slow disease progression, symptom control remains paramount to improving quality of life. Cough is a predominant symptom, however, the underlying mechanism is unknown and medications seldom provide relief (Hope-Gill et al. 2003). This study aims to characterise the way patients and their family perceive cough and the impact on their daily lives.

Methods Eligible patients had a diagnosis of IPF and a chronic cough. A convenience sample was drawn from an ongoing cross-sectional study and consented to face-to-face interviews in the outpatient setting or at home. Thematic analysis was used to analyse the transcripts.

Results 12 patients (9 male), aged 45-84, with a history of IPF spanning 1-57 months and 11 family members (females) participated. Patients and family see cough as marker of disease. Cough initially motivated patients to seek health advice which led to the diagnosis and from this, cough is perceived as a reminder of the condition and used as a measure of disease progression. There are degrees of understanding, acceptance and ability to cope with cough, depending on family members, friends and societal awareness of patients' condition. Cough also generates resentment, anger and denial, some of which stems from lack of communication.

Conclusions Patients and family use cough to monitor disease progression. However, the lack of a clear link between the two makes it challenging for clinicians to support patients to manage IPF. Communication and information sharing between patients, family and clinicians enhance coping mechanisms.

\section{WHAT ARE THE BEST SETTINGS AND METHODS FOR RECRUITMENT INTO A RANDOMISED TRIAL IN SEVERE BREATHLESSNESS?}

${ }^{1}$ Irene Higginson, ${ }^{2}$ Andrew Wilcock, ${ }^{3}$ Miriam Johnson, ${ }^{1}$ Sabrina Bajwah, ${ }^{1}$ Natasha Lovell, ${ }^{1}$ Deokhee Yi, ${ }^{4}$ Simon Hart, ${ }^{2}$ Vincent Crosby, ${ }^{5}$ Heather Poad, ${ }^{6}$ David Currow, ${ }^{7}$ Emma Best, ${ }^{7}$ Sarah Brown. ${ }^{1}$ Cicely Saunders Institute, King's College London, London, UK; ${ }^{2}$ Department of Clinical Oncology, University of Nottingham and Nottingham University Hospitals NHS Trust, Nottingham, UK; ${ }^{3}$ Wolfson Palliative Care Research Centre, Hull York Medical School, University of Hull, Hull, UK; ${ }^{4}$ Respiratory Research Group, Hull York Medical School, Castle Hill Hospital, Cottingham, UK; ${ }^{5}$ Clinical Trials Research Unit, University of Leeds, Leeds, UK; ${ }^{6}$ Faculty of Health, University of Technology, Sydney, Ultimo, New South Wales, Australia; ${ }^{7}$ Clinical Trials Research Unit, University of Leeds, Leeds, UK

10.1136/spcare-2019-mariecuriepalliativecare.33

Limited drug treatments exist to relieve severe breathlessness in advanced disease. Antidepressants warrant consideration due to potential effects. However, trials are challenging.

We aim to test the feasibility of a larger randomised mirtazapine trial, focusing on best settings and methods for recruitment. The BETter TreatmEnts for Refractory Breathlessness (BETTER-B feasibility) trial was a randomised, double-blind, placebo-controlled mixed-methods feasibility trial in London, Nottingham, Hull/York in adults with advanced diseases and modified Medical Research Council breathlessness scale (mMRC) scores of $3 / 4$.

We screened 409 patients, randomising 64 participants (30 mirtazapine, 34 placebo), averaging 1.8 patients per centre $\mathrm{pcm}$. Existing antidepressant use was the main reason for ineligibility. $63 \%$ needed help completing questionnaires. Missing data were low. Participants (47 men) had a mean age of 72 (range 51-90) years. Primary diagnoses: chronic obstructive pulmonary disease (COPD) $(40,67 \%)$, interstitial lung disease (ILD) $(19,32 \%)$, cancer (1), heart failure (4). Several had multimorbidity.

A $6.4: 1(16 \%)$ screen to recruit conversion suggests recruiting people with stage $3 / 4 \mathrm{mMRC}$ breathlessness is possible. We consider screen to recruit ratios for different settings and diagnoses. Cancer patients were difficult to recruit. Achieving recruitment requires dedicated research teams.

Six participants per arm stopped treatment prematurely; 4 mirtazapine, 5 placebo experienced serious adverse events (SAE); 1 (placebo) suspected as medication related. One 